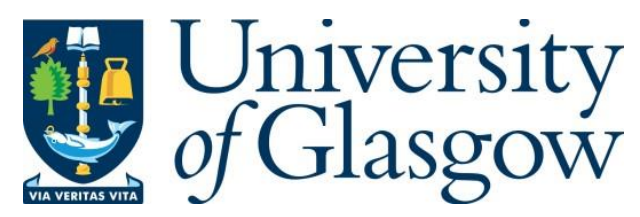

Rodrigues Lavor, I., Rabelo da Costa, D., Chaves, A., Farias, G. d. A., Macêdo, R. and Peeters, F. M. (2020) Magnetic field induced vortices in graphene quantum dots. Journal of Physics: Condensed Matter, 32(15), 155501.

There may be differences between this version and the published version. You are advised to consult the publisher's version if you wish to cite from it.

http://eprints.gla.ac.uk/207156/

Deposited on: 20 January 2020

Enlighten - Research publications by members of the University of Glasgow http://eprints.gla.ac.uk 


\title{
Magnetic field induced vortices in graphene quantum dots
}

\author{
I. R. Lavor, ${ }^{1, *}$ D. R. da Costa,${ }^{1, \dagger}$ Andrey Chaves,${ }^{1,} \ddagger$ G. A. Farias,${ }^{1}$ R. Macêdo ${ }^{2}$ and F. M. Peeters ${ }^{3,1}, \S$ \\ ${ }^{1}$ Departamento de Física, Universidade Federal do Ceará, \\ Caixa Postal 6030, Campus do Pici, 60455-900 Fortaleza, Ceará, Brazil \\ ${ }^{2}$ James Watt School of Engineering, Electronics and Nanoscale Engineering Division, \\ University of Glasgow, Glasgow G12 8QQ, United Kingdom, United Kingdom \\ ${ }^{3}$ Department of Physics, University of Antwerp, Groenenborgerlaan 171, B-2020 Antwerp, Belgium
}

(Dated: January 18, 2020)

\begin{abstract}
The energy spectrum and local current patterns in graphene quantum dots are investigated for different geometries in the presence of an external perpendicular magnetic field. Our results demonstrate that, for specific geometries and edge configurations, the quantum dot exhibits vortex and anti-vortex patterns in the local current density, in close analogy to the vortex patterns observed in the probability density current of semiconductor quantum dots, as well as in the order parameter of mesoscopic superconductors.
\end{abstract}

PACS numbers:

\section{INTRODUCTION}

In superconducting materials under an applied magnetic field, supercurrents of Cooper pairs circulate in order to expel the field, which is known as the Meissner effect. ${ }^{1}$ However, especially in superconducting alloys, if the field is greater than a certain critical value, it is able to penetrate the superconducting sample, forming localised quantized magnetic flux lines, around which a vortex is formed in the supercurrent. As the field increases, more vortices enter the sample with quantized magnetic flux, and are eventually stabilized in a triangular Abrikosov lattice. ${ }^{2-11}$

In 2003, Chibotaru and co-authors ${ }^{12}$ studied superconducting mesoscopic samples with various geometries and found that the pattern of vortices formed in these structures follows not only the formation of the Abrikosov lattice, but also the sample symmetry: if the system is a mesoscopic square, for example, vortex patterns are arranged in such squared symmetry. ${ }^{4,5,12}$ Experimentally, vortices were observed in materials ranging from: electron plasmas ${ }^{13}$ to mesoscopic superconductors with triangular ${ }^{14}$, square ${ }^{15,16}$ and circular $^{17}$ geometries. More recently, vortex patterns were also studied in artificial photonic graphene ${ }^{18}$ and thin films ${ }^{19,20}$ using optical induction method, scanning SQUID (superconducting quantum interference devices) microscopy and scanning Hall probe. respectively. However, vortices have not been observed in graphene yet. One possibility to find vortices in graphene is indirectly, using scanning tunneling microscope in order to obtain the level density of states. Other imaging techniques, like magnetic force microscopy and scanning Hall probe are also promising for visualization the magnetic fields generated by these circular currents.

Inspired by such vortex lattice formation, Slachmuylders et al. investigated a similar effect in semiconductor quantum dots (QD). ${ }^{21}$ In fact, for electrons confined in a semiconductor planar circular dot under an external magnetic field perpendicular to the system, the energy levels exhibit a series of crossings as a func- tion of field. ${ }^{21-24}$ As the angular momentum operator in this case commutes with the Hamiltonian, the energy eigenstates are also eigenstates of angular momentum, therefore, crossing points in the energy spectrum as a function of magnetic field, in fact, represent different degenerate states of the angular momentum operator. However, if the circular symmetry is broken, e.g., by a triangular or square confinement potential, the angular momentum eigenstates are no longer eigenstates of the Hamiltonian, thus enabling anti-crossings formed by states that are a combination of different angular momentum states. Moreover, in such circular geometry, Lorentz force induced by the magnetic field inside the confining geometry may be responsible for the appearance of rotating currents. Such circulating currents would be analogous to vortices in superconductors, while the angular momentum quantization is analogous to the quantization of the magnetic flux. The analogy seems to be even more justified when we realize that the first Ginzburg-Landau equation, depicting the Cooper pairs density in the superconductor, is actually the same Schrödinger equation governing electrons in the semiconductor, but with a nonlinear additional potential. ${ }^{25}$ The possibility of combining states composed by positive and negative angular momentum states at the anti-crossings of the semiconductor QD spectrum, thus allowing the existence of a network of vortices and anti-vortex, makes this an even more interesting topic. A very thorough investigation was made on these vortex states in squared and triangular semiconductor quantum dots, where a comparison to the expected vortex patterns in mesoscopic superconductors with similar geometry was performed. ${ }^{21}$

With the recent experimental discovery of graphene ${ }^{26,27}$ (for reviews, see Refs. [27-29]), there is now a new system to investigate the formation of this type of vortex. In fact, a vortex pattern in the Berry connections for a graphene quantum dot with triangular geometry was made in 2010 by Ezawa ${ }^{30}$ within continuum model. However, efforts of studying the formation of vortices induced by magnetic fields in this and other 
(a)

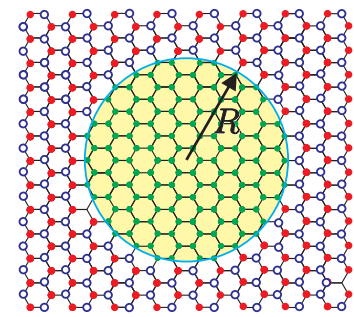

(b)

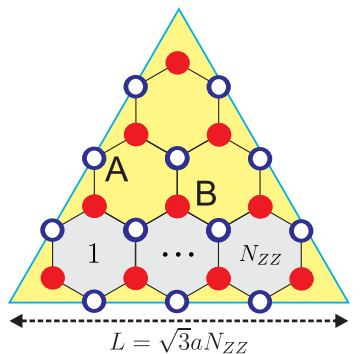

(c)

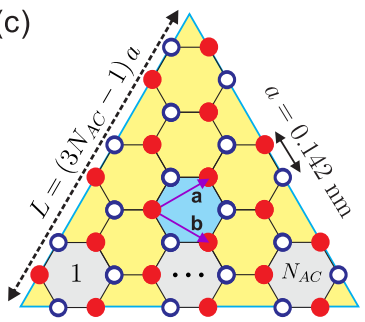

(d)

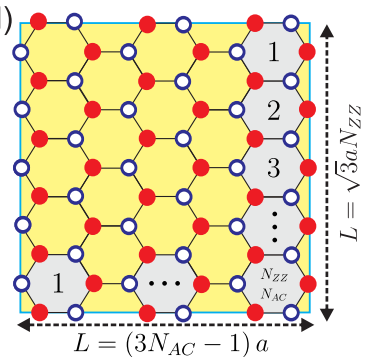

FIG. 1: (Color online) Lattice structure of (a) circular QDs, triangular QDs with (b) zigzag and (c) armchair edges, and (d) squared QDs. Primitive lattice vectors are denoted by a and $\mathbf{b}$, where $a=0.142 \mathrm{~nm}$ is the $C-C$ distance. The blue region is the area of one carbon hexagon and the atoms of the two sublattices are represented by blue (open) and red (full) dots. The side length (radius) of each structure is denoted by $L(R)$, and $N_{A C(Z Z)}$ is the number of armchair (zigzag) carbon rings in each side of the dot (in grey).

geometries and edge types are scarce in the literature. Another motivation for this study arises from the fact that, unlike usual semiconductors, ${ }^{30}$ where low-energy electrons obey the Schrödinger equation within the effective mass model, and superconductors, where Cooper pairs obey nonlinear Schrödinger equation, in graphene, the low-energy electrons obey the Dirac equation for massless particles, as we shall discuss in further detail in this work. It is then interesting to investigate whether there is indeed the formation of vortex lattices in some of the energy eigenstates of these graphene structures and, in this case, under which conditions of field strength, geometry and edge type these vortices appear, and how they relate to angular momentum states.

In this paper, we investigate, within the tight-binding (TB) model, the formation of vortex patterns in the probability density current distribution of eigenstates in graphene quantum dots, induced by the presence of a perpendicularly applied magnetic field. Different quantum dot geometries and edge types are considered, and the interplay between vortices and anti-vortices appearing in the system as the magnetic field increases is discussed in what follows.

\section{THEORETICAL MODEL}

\section{A. Probability density current}

We study monolayer graphene quantum dots (GQD) within the nearest neighbour TB model, whose Hamiltonian is given by

$$
H_{T B}=\sum_{i}\left(\epsilon_{i}+M_{i}\right) c_{i}^{\dagger} c_{i}+\sum_{\langle i, j\rangle}\left(\tau_{i j} c_{i}^{\dagger} c_{j}+\tau_{i j}^{*} c_{i} c_{j}^{\dagger}\right)
$$

where $\epsilon_{i}$ is the energy of the $i$-th site, $\tau_{i j}$ is the hopping energy $\left(\tau_{i j}=2.7 \mathrm{eV}\right), c_{i}^{\dagger}\left(c_{i}\right)$ is the creation (annihilation) operator for an electron in site $i$, and the sum is taken only between the nearest neighbors sites $i$ and $j$. Magnetic field is included via Peierls phase ${ }^{31}$ in the hopping parameters, i.e., $\tau_{i j} \rightarrow \tau_{i j} \exp \left[\frac{2 \pi i}{\phi_{0}} \int_{j}^{i} \vec{A} \cdot d \vec{l}\right]$, where $B$ is the magnetic field, $\vec{A}$ is the magnetic vector potential, and $\phi_{0}=h / e$ is the magnetic quantum flux. The energy spectra obtained in this paper are shown as a function of $\phi / \phi_{0}$, where $\phi=3 \sqrt{3} a^{2} B / 2$ is the magnetic flux through a carbon hexagon. The dot is "cut" from the original graphene flake to a desired geometry by setting a high value for $\epsilon_{i}$ (out of the energy spectrum) and $\tau_{i j}=0$ to the atoms outside the dot. It is also interesting to investigate the case where the dot is not "cut" out from the sample, but rather defined by an external region of infinite mass. Such infinite mass, described by a staggered potential $M_{i}=+V(-V)$ for sites of sublattice $A(B)$ of the honeycomb lattice of graphene, opens a gap in the energy spectrum, which avoid electrons to come out of the dot. Previous works studied theoretically the confined states by using a similar approach for graphene $^{32-39}$, bilayer ${ }^{40,41}$, and trilayer ${ }^{42}$ graphene nanostructures obtained by using the infinite mass potential. This is supposed to provide quantum confinement as much as cutting the graphene dot, but, as we will discuss latter on, edge effects play an important role in the latter case, making the energy spectrum and local current densities different as compared to the infinite mass case. We defined a very large mass-term potential of $M=0.9 \mathrm{eV}$.

The quantum dots considered in this paper has a length of the order of $10^{0} \sim 10^{1} \mathrm{~nm}$ due to computational limitations. As a result, the typical energy scale is of the order of $\mathrm{eV}$ because of the strong confinement. However, the energy scales as $E \approx \pi \hbar v_{F} / L$, with the size $L$ of the system and the magnetic field as $1 / L^{2}$. Therefore, our results can be easily be extrapolated to larger systems by making use of this scaling. The confining geometries used in this work are defined by square (SGQD), circular (CGQD), triangular zigzag (TZZGQD) and triangular armchair (TACGQD) quantum dots, as shown in Fig. 1, where zigzag and armchair refer to the edge types.

The probability density currents can be obtained, based on the method developed in Refs. [43-45], from the 
definition of probability density in the continuous space using finite difference $d f / d x=\left(f_{i}-f_{i-1}\right) / \Delta x$, so

$$
j_{i}=-\frac{\hbar}{m \Delta x} \Im\left[\Psi_{i}^{\dagger} \Psi_{i-1}\right]
$$

where $\Im[z]$ represents the imaginary part of a complex number $z$. On the other hand, using finite difference in the Schrödinger equation leads to an eigenvalue equation

$$
\begin{aligned}
E \Psi_{i}=\left(-\frac{\hbar^{2}}{2 m \Delta x^{2}}\right) \Psi_{i+1} & +\left(-\frac{\hbar^{2}}{m \Delta x^{2}}\right) \Psi_{i} \\
& +\left(-\frac{\hbar^{2}}{2 m \Delta x^{2}}\right) \Psi_{i-1}
\end{aligned}
$$

which is equivalent to a one-dimensional first nearest neighbors TB model, where the hopping is $\tau=$ $-\hbar^{2} / 2 m \Delta x$ and $\Delta x$ is equivalent to the distance between the neighbors. Therefore, following this analogy, we rewrite Eq. (2) as

$$
j_{i}=-\frac{2 a}{\hbar} \Im\left[\Psi_{i}^{\dagger} \Psi_{i-1} \tau\right]=-\frac{2 a}{\hbar} \Im\left[\Psi_{i}^{\dagger} \Psi_{i-1} H_{i, i-1}\right] .
$$

By defining the location of the sites through lines $(i)$ and columns $(j)$ in the graphene lattice, we obtain ${ }^{44-46}$

$$
\begin{aligned}
& j_{x}(i, j)= \pm \frac{a}{\hbar}\left\{2 \Im\left[\Psi_{i, j} \Psi_{i, j \pm 1}^{\dagger} \tau_{i, j \pm 1}\right]\right. \\
& \left.-\Im\left[\Psi_{i, j} \Psi_{i-1, j}^{\dagger} \tau_{i-1, j}\right]-\Im\left[\Psi_{i, j} \Psi_{i+1, j}^{\dagger} \tau_{i+1, j}\right]\right\}
\end{aligned}
$$

and

$$
\begin{aligned}
& j_{y}(i, j)=\frac{\sqrt{3} a}{\hbar}\left\{\Im\left[\Psi_{i, j} \Psi_{i+1, j}^{\dagger} \tau_{i+1, j}\right]\right. \\
&\left.-\Im\left[\Psi_{i, j} \Psi_{i-1, j}^{\dagger} \tau_{i-1, j}\right]\right\}
\end{aligned}
$$

where the $\mp \operatorname{sign}$ in $j_{x}$ will be positive (negative) if the $(i, j)$ site belongs to the sublattice $A(B)$.

\section{B. Energy spectrum of a CGQD revisited}

In order to understand the energy states and current distributions in GQDs, it is pedagogical to start with an analysis of the eigenstates of a CGQD [Fig. 1(a)] under a perpendicular magnetic field, which have been analytically calculated in previous studies ${ }^{32-34}$ from the Dirac equation for low energy electrons in graphene by applying Berry and Mondragon ${ }^{47}$ boundary condition, that was investigated half a century ago in the context of confinement of neutrino's, and also numerically computed by using TB model as reported in Refs. [33,48-50]. It is relevant to mention that another route to find the CGQD energy states could be used, as discussed in Ref. [37], whose analytical derivation is based on the scattering problem formalism to obtain the boundary conditions due to a circular mass barrier in monolayer graphene. We follow the description used in Refs. [33,34] and only the most important steps of these calculations will be repeated here for completeness.

We assume a Dirac Hamiltonian, which describes the low energy states of the charge carriers located in the vicinity of the Dirac valley $K$, with a mass-related term $M(\vec{r})$, given by

$$
H=v_{f}(\vec{p}+e \vec{A}) \cdot \vec{\sigma}+M(\vec{r}) \sigma_{z},
$$

and the Dirac equation is $H \psi(r, \theta)=E \psi(r, \theta)$, with the wave function being a two-component spinor $\psi(r, \theta)=$ $\left[\psi_{1}(r, \theta), \psi_{2}(r, \theta)\right]^{T}$. Here, the Fermi velocity is $v_{f}$, $e$ is the absolute value of the electron charge, $\vec{A}=$ $\frac{B}{2} r(-\sin \theta, \cos \theta, 0)$ is the symmetric gauge for the vector potential, with $\theta$ being the polar angle, and $\vec{\sigma}=\left(\sigma_{x}, \sigma_{y}\right)$ are the Pauli's spin matrices in the basis of the two sublattices $A$ and $B$. We assume that the electrons are confined only in the circular region, which is modeled by an infinite mass outside of the QD, i.e., $M(\vec{r})=0$ for $r<R$ and $M(\vec{r}) \rightarrow \infty$ for $r \geq R$, where $r$ is the radial coordinate and $R$ the QD radius. As a consequence, we have an infinite mass boundary condition, so that $\psi_{2} / \psi_{1}=i \zeta \exp (i \theta) .{ }^{47}$ Note that we are solving this problem by taking the valley-isotropic format of the Dirac Hamiltonian, it means that, although we use the Dirac Hamiltonian that holds for the $K$ valley states the valleys are now differentiated by the boundary condition quantum number $\zeta$, that takes values $+1(-1)$ for $K\left(K^{\prime}\right)$ valley.

For $r<R$, Eq. (6) reduces to $H=v_{f}(-i \hbar \vec{\nabla}+e \vec{A}) \cdot \vec{\sigma}$. In cylindrical coordinates, $\nabla=\hat{r} \partial_{r}+\frac{1}{r} \hat{\theta} \partial_{\theta}$ and writing unit vectors as $\hat{r}=\cos \theta \hat{x}+\sin \theta \hat{y}$ and $\hat{\theta}=-\sin \theta \hat{x}+\cos \theta \hat{y}$, the Hamiltonian can be rewritten as

$$
H=-i \hbar v_{f}\left(\begin{array}{cc}
0 & e^{-i \theta}\left[\partial_{r}-\frac{i}{r} \partial_{\theta}+\frac{e B r}{2 \hbar}\right] \\
e^{i \theta}\left[\partial_{r}+\frac{i}{r} \partial_{\theta}-\frac{e B r}{2 \hbar}\right] & 0
\end{array}\right)
$$

Since the Hamiltonian described by Eq. (7) commutes with the total angular momentum operator $J_{z}=l_{z}+$ $\hbar \sigma_{z} / 2$, i.e., $\left[H, J_{z}\right]=0$, we take simultaneous eigenstates of $H$ and $J_{z}$ :

$$
\psi(r, \theta)=e^{i m \theta}\left(\begin{array}{c}
\chi_{1}(r) \\
e^{i \theta} \chi_{2}(r)
\end{array}\right)
$$

where $m=0, \pm 1, \pm 2, \pm 3, \cdots$ is the total angular momentum quantum number.

Substituting Eqs. (7) and (8) in the Dirac equation and introducing the magnetic length as $l_{B}=\sqrt{\hbar / e B}$, we obtain the following decoupled second order differential equation

$$
\left[\partial_{r}^{2}+\frac{1}{r} \partial_{r}-\frac{(m+1)}{l_{B}^{2}}-\frac{m^{2}}{r^{2}}-\frac{r^{2}}{4 l_{B}^{4}}+\left(\frac{E}{\hbar v_{f}}\right)^{2}\right] \chi_{1}(r)=0 .
$$

In order to solve Eq. (9), let's assume the ansatz $\chi_{1}(r)=$ $r^{m} \exp \left[-r^{2} / 4 l_{B}^{2}\right] \xi\left(r^{2} / 2 l_{B}^{2}\right)$, which generates the follow- 


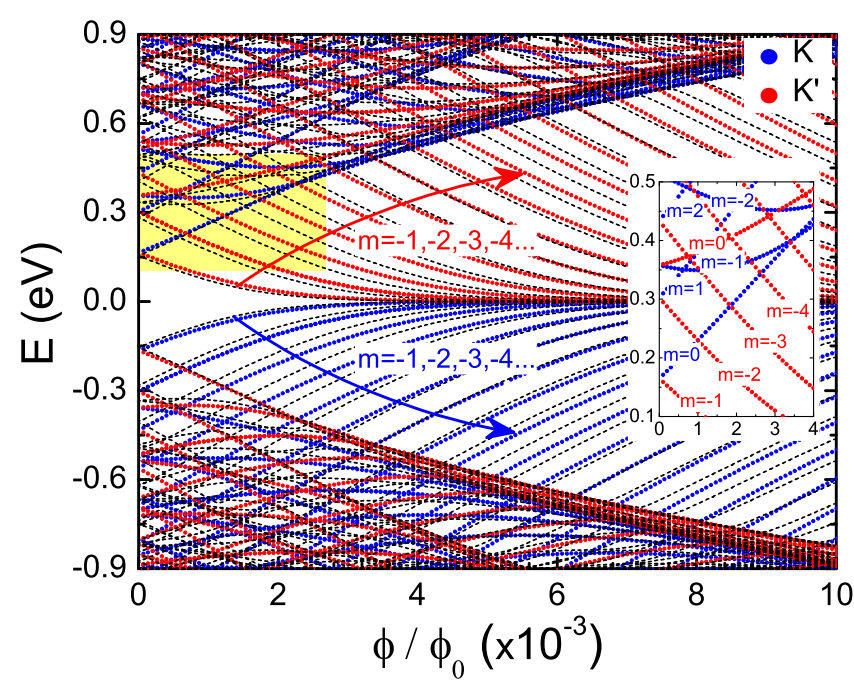

FIG. 2: (Color online) Energy spectrum of CGQD with radius $R=50 \mathrm{~nm}$ as a function of the magnetic flux calculated by using (black dotted lines) TB and (circular symbols) continuum models for the (blue) $K$ and (red) $K^{\prime}$ valleys. The inset shows an enlargement of the yellow shaded area for the low energy levels at small magnetic fields.

ing confluent hypergeometric ordinary differential equation

$$
\left\{\widetilde{r} \partial_{\widetilde{r}}^{2}+(m+1-\widetilde{r}) \partial_{\widetilde{r}}-\left[m+1-\frac{1}{2}\left(\frac{E l_{B}}{\hbar v_{f}}\right)^{2}\right]\right\} \xi(\widetilde{r})=0,
$$

with $\tilde{r} \equiv r^{2} / 2 l_{B}^{2}$. The solutions are the confluent hypergeometric function of the first kind ${ }_{1} F_{1}(\alpha, m+1, \widetilde{r})$ or also called Kummer function $M(\alpha, m+1, \widetilde{r})$, with $\alpha \equiv m+1-\left(E l_{B} / \hbar v_{f}\right)^{2} / 2$. However, since $m$ can assume negative integer values, it is more appropriate to take the regularized confluent hypergeometric function, which satisfies the same differential equation (10) but with a more general argument, defined as $\widetilde{M}(\alpha, m+1, \widetilde{r})=$ $M(\alpha, m+1, \widetilde{r}) / \Gamma(m+1) .{ }^{51}$ Therefore, we have that

$$
\psi_{1}(r, \theta)=C e^{i m \theta} r^{m} e^{-r^{2} / 4 l_{B}^{2}} \widetilde{M}\left(\alpha, m+1, \frac{r^{2}}{2 l_{B}^{2}}\right),
$$

where $C$ is the normalization constant. The component $\psi_{2}$ is found by inserting the solution for $\psi_{1}$ in the differential equation coming from Eq. (7). Using the properties of the regularized confluent hypergeometric function ${ }^{52}$, this results into

$$
\psi_{2}(r, \theta)=\frac{i C E}{2 \hbar v_{f}} e^{i(m+1) \theta} r^{m+1} e^{-r^{2} / 4 l_{B}^{2}} \widetilde{M}\left(\alpha, m+2, \frac{r^{2}}{2 l_{B}^{2}}\right) .
$$

Using the infinite mass boundary condition at $r=R$, that gives $\chi_{2}(R) / \chi_{1}(R)=i \zeta$, and from Eqs. (11) and (12), we obtain the following transcendental equation that allows us to determine the energy levels

$$
\frac{\zeta E}{2 \hbar v_{f}} \widetilde{M}\left(\alpha, m+2, \frac{r^{2}}{2 l_{B}^{2}}\right)-\widetilde{M}\left(\alpha, m+1, \frac{r^{2}}{2 l_{B}^{2}}\right)=0 .
$$

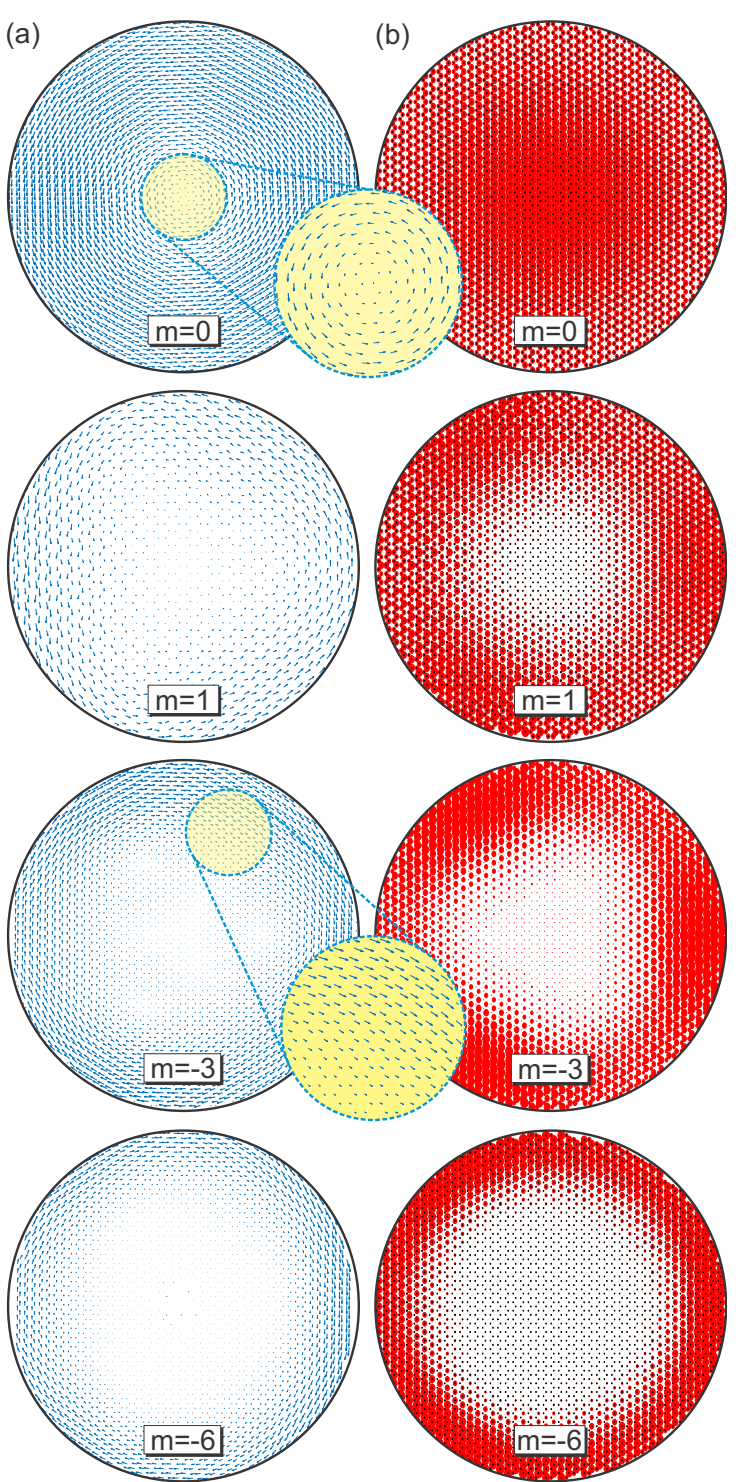

FIG. 3: (Color online) (a) Local density currents and (b) probability densities in a CGQD, along with a zoom in a specific region of the QD to emphasize the current pattern for states $m=0,+1,-2$ and -6 . The currents are composed of almost concentric rings of current flowing in the clockwise (counterclockwise) direction if the value of $m$ is negative (positive).

Since the regularized confluent hypergeometric functions are oscillatory functions, there is an infinite number of $E_{n}^{\prime}$ s for given $B, m$, and $\zeta$ which satisfy the above equation. This defines the radial quantum number $n$, that is also identified as the Landau level number. Thus, one labels the roots of Eq. (13) by three indices: $(n, m, \zeta) \equiv$ (the principal quantum number, the total angular quantum number, the valley index).

The advantage of having such analytical expression for the eigenenergies lies on the possibility of identifying the angular momentum nature of the eigenstates obtained from the numerical TB approach. In cases where 
(a)

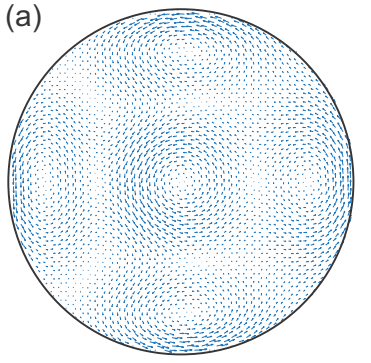

(b)

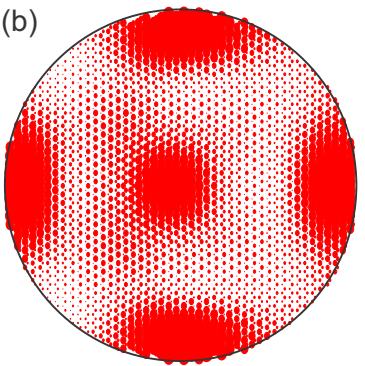

FIG. 4: (Color online) (a) Probability density current pattern and (b) probability density obtained by the TB model for the state with $\phi / \phi_{0}=1.0 \times 10^{-3}$ and $E \approx 0.496 \mathrm{eV}$ in Fig. 2 .

the circular symmetry is broken, such as in triangular and square dots, the eigenstates are no longer expected to exhibit simply circular currents along the dot edges, ${ }^{32-39,48-50,53-55}$ but the analytical solution given here still provides important information on the vortex/antivortex pattern expected for these geometries, as we will see further.

\section{RESULTS AND DISCUSSION}

\section{A. CGQDs}

Figure 2 illustrates a comparison between the analytical solution (symbols) obtained from Eq. (13) and the energy spectrum obtained from the TB model (dashed black lines) for charge carriers confined in a CGQD as a function of magnetic field. Blue and red dots represent analytical results for the $K$ and $K^{\prime}$ valleys, respectively. An intervalley electron-hole symmetry between states of the same $n$ and $m$ is observed, i.e. $-E(n, m, \zeta)=$ $E(n, m,-\zeta)$, as pointed out in previous works for both zero and nonzero magnetic fields cases. ${ }^{32-34}$ The analytical spectrum allows one to assign values of angular momentum $m$ to each eigenstate.

From the numerical CGQD eigenfunctions computed via TB model, we plot in Fig. 3 the (a) local density currents and (b) probability densities for states assigned equivalently to total angular momentum index $m=0,+1,-3$ and -6 in the continuum model, for further comparison with those of TZZGQD, TACGQD and SGQD. The same value of magnetic flux $\phi / \phi_{0}=$ $7.5 \times 10^{-4}$ is used in all cases, except for $m=-6$, whose numerical state is harder to isolate from the other states in the energy spectrum, and therefore we took the flux $\phi / \phi_{0}=3.0 \times 10^{-3}$. We verify that as the magnetic field increases, for the same state $m$, the current densities shown in Fig. 3(a) just become more concentrated towards the perimeter of the circular dot, as a consequence of the fact that the radius of an electron in a circular orbit due to a perpendicular magnetic field is inversely proportional to the field intensity.

From the continuum model of a CGQD discussed in

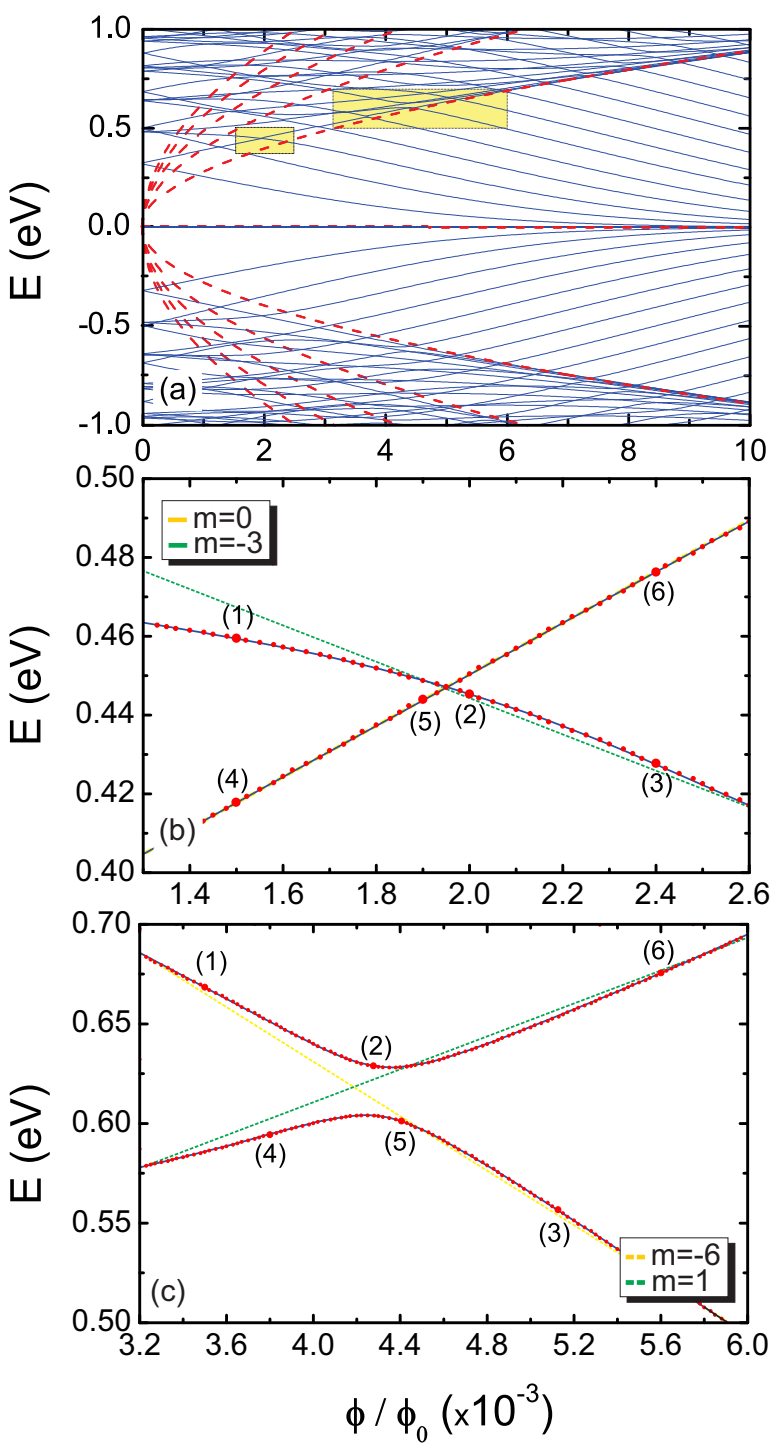

FIG. 5: (Color online) (a) Energy spectrum of a TZZGQD, with $N_{Z Z}=50$ carbon rings in each of its sides, as a function of the magnetic flux through a single carbon hexagon. Red lines are the Landau levels obtained from the Dirac model for an infinite graphene layer. Sectors of this energy spectrum (yellow shaded region) emphasizing crossings and anticrossings are shown in (b) and (c), respectively. Current flow maps and probability density current patterns for the states labelled as (1)-(6) around the crossing and anti-crossing points are shown in Figs. 6(a) and (b), respectively. Solid and dashed lines in (b) and (c) correspond, qualitatively, to the analytical energy levels of the CGQD obtained within the continuum model, for different values of angular momentum index $m$. Only the states that will be discussed in the text are shown in (b) and (c), in order to make the crossing and anti-crossing states more evident.

Sec. II B, it is expected that all currents are composed of concentrics rings of current flowing in the clockwise (counterclockwise) direction if the value of $m$ is negative (positive). ${ }^{33}$ However, due to the hexagonal geometry of the graphene crystal lattice, it is not possible to obtain 


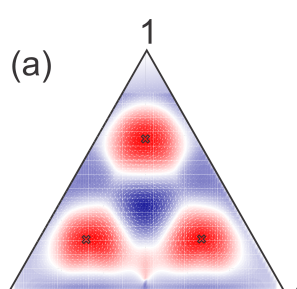

$\mathrm{m}=-3$

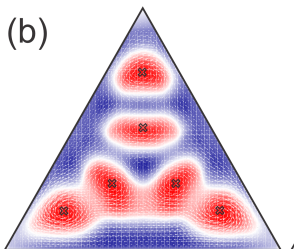

$m=-6$

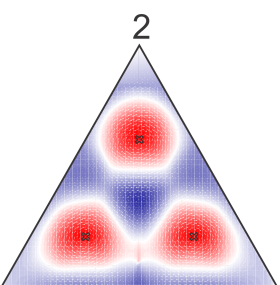

$\mathrm{m}=-3$

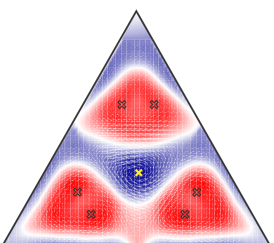

$(-3)$

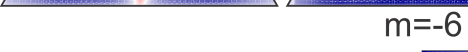

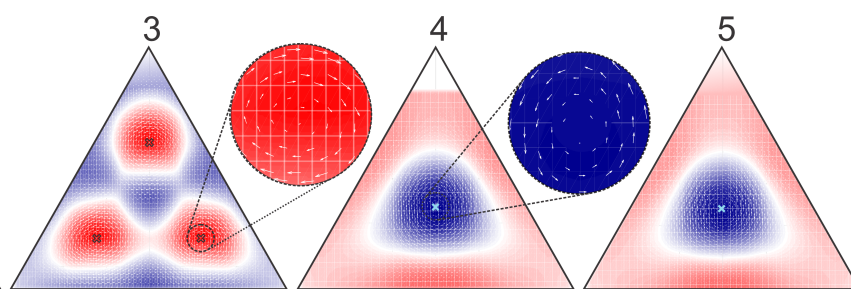

$\mathrm{m}=0$
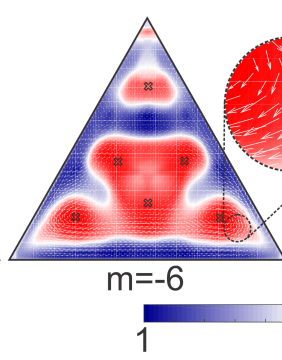

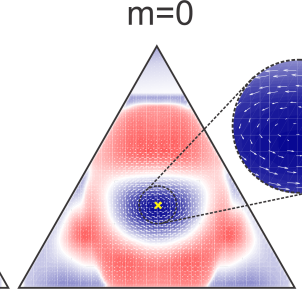

$\mathrm{m}=+1$

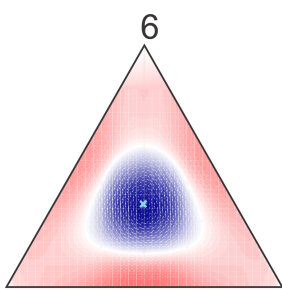

$m=0$

FIG. 6: (Color online) Vortex locations (black, blue and yellow crosses, with $m=-1, m=0$ and $m=+1$, respectively, where $m$ correspond to the value of angular momentum), probability density currents (arrows), and current flow (color map) in a TZZGQD for states involved in (a) crossing and (b) anti-crossing points of the energy spectrum, labelled as (1) to (6) in Figs. 5(b) and (c). The current flow scale ranges from -1 , for clockwise flow (red), to +1 , for counterclockwise flow (blue).

a perfectly CGQD. Therefore, some confined states obtained from the TB model exhibit non-concentric current flows. An example of such current pattern is illustrated in Fig. 4, for the state with energy $E \approx 0.496 \mathrm{eV}$ under a $\phi / \phi_{0}=1.0 \times 10^{-3}$ magnetic flux. This unexpected result, which is not captured by a continuum Dirac model, can only be described in a TB approach, draws attention to the possibility of observing vortices rather than the usual circular currents in a GQD this will be even more so when the circular symmetry is broken, as in a square or a triangle.

\section{B. TZZGQDs}

The geometry and energy spectrum of TZZGQD are illustrated in Figs. 1(b) and 5(a), respectively. The dashed red lines in the latter show the lowest Landau levels in the Dirac model for an infinite graphene layer, for comparison. The magnetic levels in the QD converge to the Landau levels as the magnetic field increases due to the fact that the magnetic length becomes smaller than the system size, so that confinement effects are strongly reduced. The number of carbon rings along the side of this structure is $N_{Z Z}$, and the side length is given by $L_{Z Z}=\sqrt{3} a N_{Z Z}$, where $a=0.142 \mathrm{~nm}$ is the distance between adjacent carbon atoms. We considered $N_{Z Z}=50$ that corresponds to $L \approx 12.3 \mathrm{~nm}$. A careful analysis of Fig. 5(a) reveals crossings and anti-crossings between states as the magnetic field increases, as emphasized in Figs. 5(b) and 5(c), respectively, where magnifications of two different shaded yellow regions of the result in Fig. 5(a) are shown.

The probability density currents illustrated in Fig. 6(a) are obtained from the wave functions corresponding to each energy state labelled from (1) to (6) in Fig. 5(b) for the crossing situation. They exhibit clear vortex patterns that do not change along a given line of states $(1-3$ or $4-6$ ) and obey the symmetry of the QD. In order to identify the vortex patterns and locations in a more systematic way, we made use of a vortex core identification algorithm (for more details, see Ref. [56]). The algorithm also identifies current flows by a parameter between -1 and +1 , where $-1(+1)$ represents a current flow in the clockwise (counterclockwise) direction. From now onwards, all current patterns will be plotted along with a color map identifying current flow direction.

For the state (1) in Fig. 6(a), there are three vortices in clockwise direction, i.e., with $m=-1$ (black crosses), in a pattern that remains the same up to state (3). The total value of angular momentum of this state is roughly equal to the sum of each $m$ in its respective configuration, so, for these three states, the total angular momentum index is -3 . On the other hand, there is only one vortex in the counterclockwise direction for states (4)-(6), with $m=0$ (blue cross). One can compare the latter to the current pattern of a $m=0$ state in a CGQD shown in Fig. 3(a), for example.

It is also noted that states whose energy decreases with increasing magnetic field [(1)-(3) in Figs. 6(a) and 6(b)] have negative overall angular momentum, whereas states with increasing energy as a function of the magnetic field [(4)-(6) in Figs. 6(a) and 6(b)], have zero or positive overall angular momentum. This statement can be also verified in the inset of Fig. 2 for the CGQD.

An interesting phenomenum occurs with the energy states which form an anti-crossing, which arises due to a break in the otherwise circular symmetry of the QD. Figure 5(c) shows such an anti-crossing, whose respective vortices are shown in Fig. 6(b). We observe a clear change 
in the vortex pattern, through the creation/annihilation of vortices, as the magnetic field increases. The color map helps us to identify such changes in the vortex pattern, where one observes vortices leaving and entering the sample. States (1) and (3) both exhibit six vortices in clockwise direction, i.e., with $m=-1$. The total angular momentum index is then -6 for both. For the states (4) and (6), however, we see only one vortex in counterclockwise direction $(m=+1)$. In this case, the total angular momentum index is +1 . The vortex pattern becomes more defined for states that are further away from the anti-crossing.

The full (green) and dashed (yellow) lines in Figs. 5(b) and 5(c) correspond, qualitatively, to the energy levels of the CGQD (Fig. 2) with the same values of $m$ as discussed here, namely, $m=-3$ and 0 in the former, and $m=-6$ and +1 in the latter. At the anti-crossing point, the vortex pattern seems mixed: following the higher energy states line, an extra counterclockwise vortex at the center of the QD is added to the pattern in (1) to form (2), whereas the other six clockwise vortices leave the system to form (6). Similarly, but now for the lower energy states, the clockwise currents (red) approach the counterclockwise vortex in (4) to form (5). As the magnetic field increases further, these clockwise currents starts to dominate and state (3) is formed.

\section{TACGQDs}

The energy spectrum as function of magnetic flux for a TACGQD (see Fig. 1(c)) with $N_{A C}=35$ is depicted in Fig. 7(a). A magnification on a particular region of the spectrum, where an anti-crossing occurs, is shown in Fig. 7(b). The side length in this case is given by $L_{A C}=\left(3 N_{A C}-1\right) a$, so that the dot side is approximately $15.2 \mathrm{~nm}$.

The probability current patterns in the states labelled by (1)-(4) in Fig. 7(b) are illustrated in Fig. 8. The vortex and current flow pattern illustrated in Fig. 8 reflects again the triangular symmetry of the GQD and resembles the patterns in TZZGQD: we observe a clear change in the vortex structure, throught the creation/annihilation of vortices from states (1)-(4). For (1) and (2) in Fig. 8, we observe six vortices in clockwise direction, and the total angular momentum is -6 . On the other hand, for the configurations (3) and (4), the total angular momentum is +1 , as there is only one vortex in the counterclockwise direction. Therefore, although lying in different branches of the energy spectrum, it is clear that states (3) and (4) (or, equivalently, (1) and (2)) represent, qualitatively, states with vortex patterns of the same nature.

\section{SGQDs}

Similar conclusions can be drawn when assuming a QD with square symmetry, such as Fig. 1(d). For this
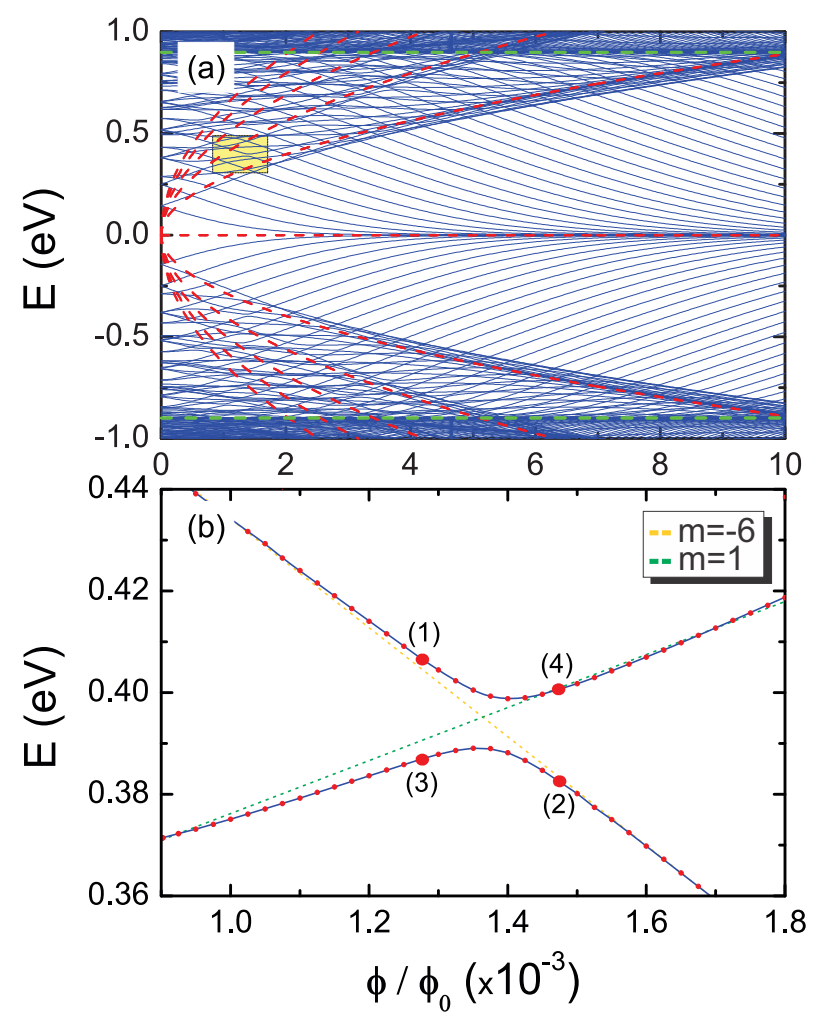

FIG. 7: (Color online) (a) Energy spectrum of a TACGQD, with $N_{A C}=35$ carbon rings in each of its sides, as a function of the magnetic flux through a single carbon hexagon. The red lines are Landau levels of an infinite graphene layer, shown here just for reference. The yellow shaded sector of this energy spectrum, emphasizing an anti-crossing of energy states, is magnified in (b). Current flow maps and probability density current patterns for the states labelled as (1)-(4) around the anti-crossing are shown in Fig. 8. Dashed lines in (b) correspond, qualitatively, to the analytical energy levels of the CGQD obtained within the continuum model, for different values of angular momentum index $m$. Only the states that will be discussed in the text are shown in (b), in order to make the anti-crossing states more evident. The green dashed lines in (a) correspond to the assumed mass potential value in the dot boundary definition $(M=0.9 \mathrm{eV})$.

structure, we consider $N_{A C}=23$ and $N_{Z Z}=34$, so that the dot length is approximately $L_{A C} \approx 9.7 \mathrm{~nm}$ $\left(L_{Z Z} \approx 8.4 \mathrm{~nm}\right)$ for the armchair (zigzag) side. The energy spectrum of such SGQD as a function of the magnetic flux is shown in Fig. 9(a). A magnification on a particular (yellow shaded) region of this spectrum, where an anti-crossing occurs, is shown in Fig. 9(b). The current flow map and vortex locations for the states (1)-(4) in Fig. 9(b) are illustrated in Fig. 10. Again, one observes a change in the vortex pattern as the magnetic field increases and the color map helps us to identify an asymmetry on the position of the vortices with respect to the vertical axis. This asymmetry simply reflects the asymmetry of the mass potential surrounding the square, which can not be made symmetrical in the case of SGQD, 

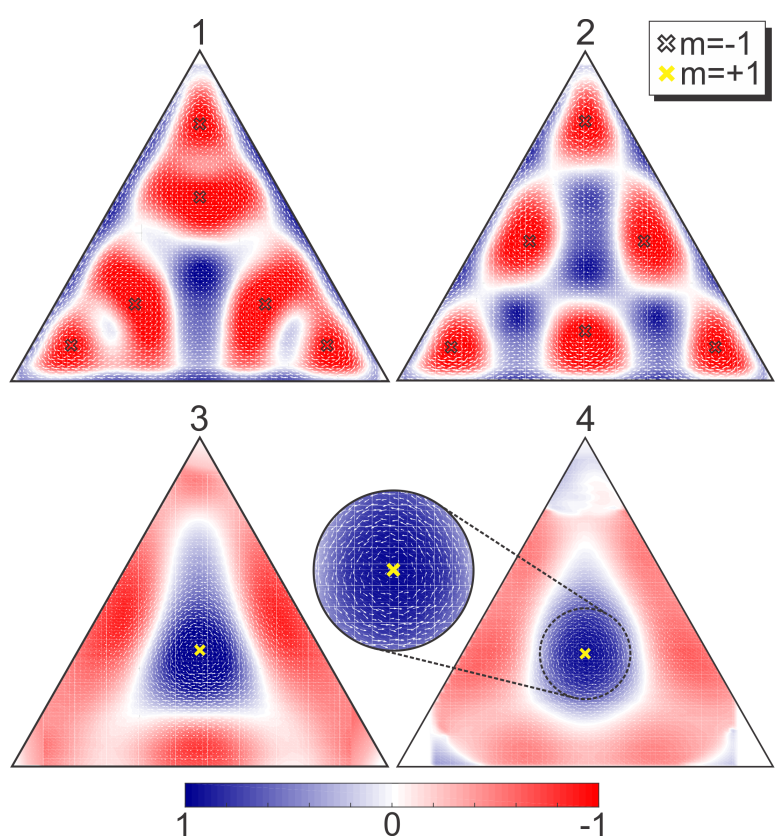

FIG. 8: (Color online) Vortex locations (black and yellow crosses, with $m=-1$ and $m=+1$, respectively, where $m$ correspond to the value of angular momentum) represents a current flow in the clockwise (counterclockwise) direction), probability density currents (arrows), and current flow (color map) in a TACGQD, for the states labelled as (1)-(4) involved in the anti-crossing shown in Fig. 7(b). The current flow scale ranges from -1 , for clockwise flow (red), to +1 , for counterclockwise flow (blue).

since the left and right sides of this dot are composed of zigzag edges of atoms belonging to different sub-lattices (see Fig. 1(d)), for which the assigned potentials $M_{i}$ are opposite.

For the states labelled as (1) and (2) in Fig. 10, we observe six vortices winding in clockwise direction $(m=-1)$, consequently, the overall angular momentum index is equal to -6 . On the other hand, for the states (3) and (4), only one vortex exists, and it winds towards the counterclockwise direction, with $m=0$. It is thus clear, following the upper energy line in Fig. 9(b), that clockwise vortices in state (1) move away from the sample as the magnetic field increases, while counterclockwise vortices enter the sample from the borders to form the single counterclockwise vortex, in the center of the sample, in state (4). Similarly, following the lower energy branch in Fig. 9(b), one observes the clockwise vortices in the borders of the sample dominate over the central counterclockwise vortex in state (3) to eventually form state (2).

\section{CONCLUSIONS}

We investigated vortex patterns in the probability density currents of electrons in GQDs, induced by an homo-
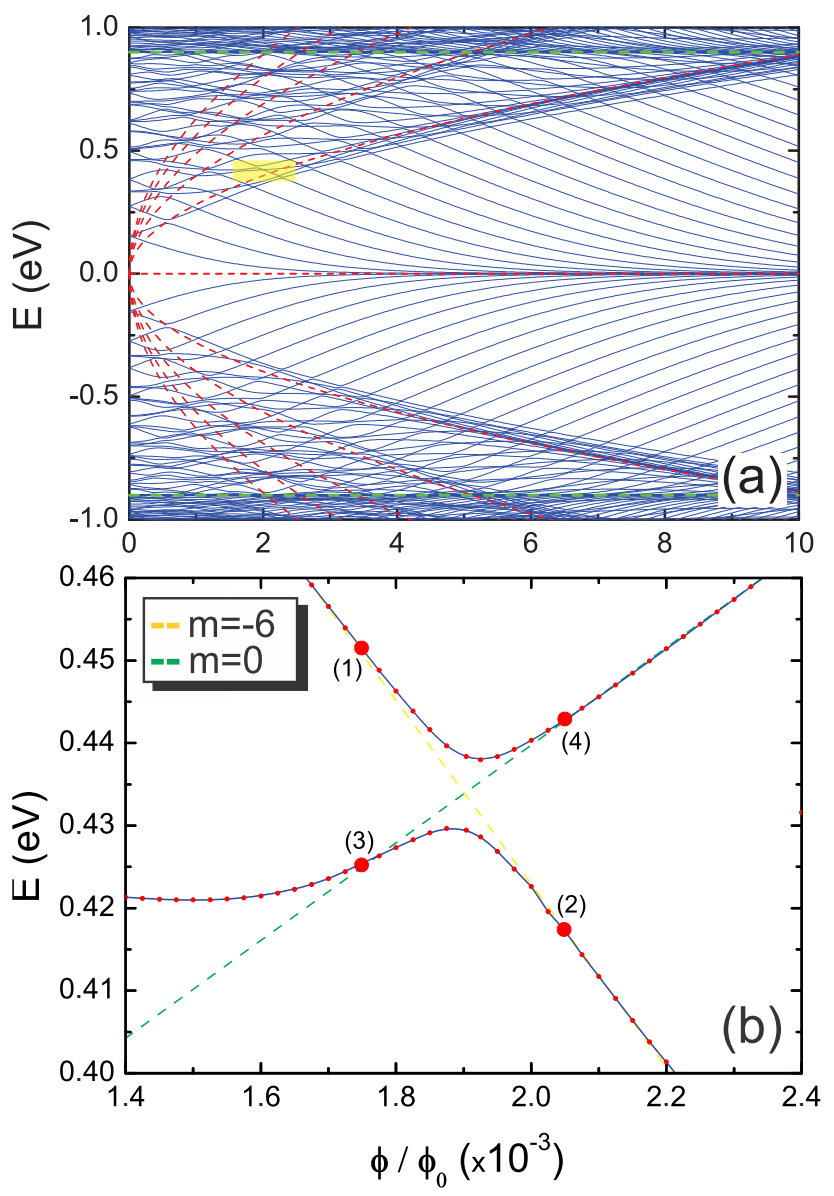

FIG. 9: (Color online) (a) Energy spectrum of a SGQD, with $N_{A C}=23$ and $N_{Z Z}=34$ carbon rings in each side, as a function of the magnetic flux through a single carbon hexagon. The red lines are Landau levels of an infinite graphene layer, shown here just for reference. The shaded (yellow) sector of this energy spectrum, emphasizing an anti-crossing, is magnified in (b). Flux flow maps and probability density current patterns for the states labelled as (1)-(6) around the anticrossings are shown in Fig. 10. Dashed lines in (b) correspond, qualitatively, to the analytical energy levels of the CGQD obtained within the continuum model, for different values of angular momentum index $m$. Only the states that will be discussed in the text are shown in (b), in order to make the anti-crossing states more evident. The green dashed lines in (a) correspond to the assumed mass potential value in the dot boundary definition $(M=0.9 \mathrm{eV})$.

geneous perpendicularly applied external magnetic field. For this purpose, we used a nearest neighbors TB method and a vortex core identification algorithm. The effect of the geometry (circular, squared and triangular) of the QDs, with zigzag and armchair edge types, on the current profile was discussed. From a comparison between the TB results for QDs with different geometries and analytical results for the specific case of a circular dot, it is possible to identify the $K-K^{\prime}$ valley nature and the total angular momentum for each eigenstate of the dot. This provides information on the number of vortices that may 


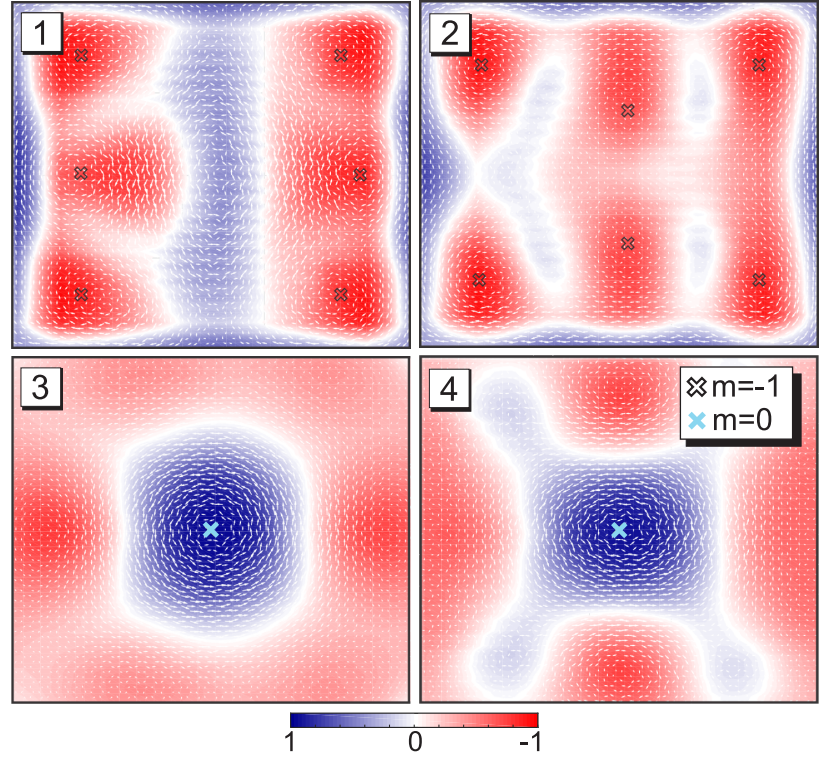

FIG. 10: (Color online) Current flow (color map), probability density currents (arrows) and vortex localizations (black and blue crosses, with $m=-1$ and $m=0$, respectively, where $m$ correspond to the value of angular momentum) in a SGQD, for states labelled as (1)-(4) in Fig. 9(b). The current flow scale ranges from -1 , for clockwise flow (red), to +1 , for counterclockwise flow (blue).

appear in the probability density currents of these dots as the magnetic field increases.

In an ideal CGQD, in a continuum model, angular momentum is a good quantum number and the energy spectrum thus exhibits crossings between energy states with different angular momentum index as the magnetic field increases. However, when atomic-scale details of the dot edges are taken into account in a TB model, our results shows that even for a circular geometry, the energy spectrum exhibit anti-crossings, which are more evident as the size of the CGQD decreases, as a consequence of the fact that imperfections in the otherwise circular dot geometry become more evident for small QDs. Vortex patterns are already observed in such CGQD, which is not predicted by the continuum model. As the circular geometry is broken further, into a triangle or square, anti-crossings of energy levels become even more evident.

Anti-crossings between energy states as the magnetic field increases are seen as a consequence of the fact that, as the circular symmetry of the dot is broken, angular momentum states are no longer good quantum numbers. As a result, different angular momentum states may be combined to form eigenstates of the system for triangular and square geometries. Probability density current patterns follow the dot geometry, so that the different angular momentum states in these systems manifest themselves in the form of vortices, distributed across the dot. In an ideal CGQD, the vorticity would correspond to the quantized angular momentum states, so that $\left\langle L_{z}\right\rangle / \hbar$ is always an integer number and probability density currents always consists of concentric clockwise and counterclockwise flows. However, for square and triangular geometries, $\left[H, L_{z}\right] \neq 0$ and, as a consequence, $\left\langle L_{z}\right\rangle$ varies continuously as the magnetic field increases, thus making non-concentric clockwise and counterclockwise vortices enter/exit at the sample, exactly along the anti-crossings in the energy spectrum, where non-integer values of $\left\langle L_{z}\right\rangle / \hbar$ are expected.

Results presented here demonstrate that the appearance of vortices in mesoscopic systems under external magnetic fields is not an exclusive feature of Schrödingerlike quasi-particles (i.e. electrons and holes) in semiconductor dots, or Cooper pairs in superconducting systems, but is also present for the Dirac-like low energy electrons in GQDs. Finally, we hope this theoretical prediction will inspire works for looking at the experimental evidence of vortex pattern formation in graphene quantum dots.

\section{Acknowledgments}

This work was financially supported by the CAPES foundation and $\mathrm{CNPq}_{\text {(Science Without Borders, } \mathrm{PQ} \text { and }}$ FUNCAP/PRONEX programs).
* Electronic address: icaro@fisica.ufc.br

$\dagger$ Electronic address: diego_rabelo@fisica.ufc.br

¥ Electronic address: andrey@fisica.ufc.br

$\S$ Electronic address: francois.peeters@ua.ac.be

1 J. Bardeen, L. N. Cooper, and J. R. Schrieffer. Theory of Superconductivity. Phys. Rev. 108, 1175 (1957).

${ }^{2}$ W. Wu, H. J. Zhao, B. Li, and Z. X. Shi. Finite-size and shape effects on vortex configurations in superconducting isosceles right triangles. Supercond. Sci. Technol. 30, 125004 (2017).

${ }^{3}$ H. J. Zhao, V. R. Misko, F. M. Peeters, S. Dubonos, V. Oboznov, and I. V. Grigorieva. Vortex configurations in mesoscopic superconducting triangles: Finite-size and shape effects. Europhys. Lett. 83, 17008 (2008).
${ }^{4}$ B. J. Baelus and F. M. Peeters. Dependence of the vortex configuration on the geometry of mesoscopic flat samples. Phys. Rev. B 65, 104515 (2002).

${ }^{5}$ F. M. Peeters, V. A. Schweigert, B. J. Baelus, and P. S. Deo. Vortex matter in mesoscopic superconducting disks and rings. Physica C 332, 255 (2000).

${ }^{6}$ B. Chen, W. P. Halperin, P. Guptasarma, D. G. Hinks, V. F. Mitrović, A. P. Reyes, and P. L. Kuhns. Twodimensional vortices in superconductors. Nat. Phys. 3, 239 (2007).

7 G. Blatter, M. V. Feigel'man, V. B. Geshkenbein, A. I. Larkin, and V. M. Vinokur. Vortices in high-temperature superconductors. Rev. Mod. Phys. 66, 1125 (1994).

8 J. Deang, Q. Du, M. Gunzburger, and J. Peterson. Vortices 
in superconductors: Modelling and computer simulations. Phil. Trans. R. Soc. Lond. A 355, 1957 (1997).

${ }^{9}$ E. H. Brandt. Vortices in superconductors: Ideal lattice, pinning, and geometry effects. Supercond. Sci. Technol. 22, 034019 (2009).

10 A. Tonomura. Direct observation of vortices in superconductors by using a field-emission electron microscope. JSAP International 2, 4 (2000).

${ }^{11}$ M. V. Milošević and F. M. Peeters. Vortex-Antivortex Lattices in Superconducting Films with Magnetic Pinning Arrays. Phys. Rev. Lett. 93, 267006 (2004).

12 L. F. Chibotaru, A. Ceulemans, M. Lorenzini, and V. V. Moshchalkov. Vorticity quantum numbers for confined electrons. Europhys. Lett. 63, 159 (2003).

13 C. F. Driscoll and K. S. Fine. Experiments on vortex dynamics in pure electron plasmas. Phys. Fluids B: Plasma Physics 2, 1359 (1990).

${ }^{14}$ L. F. Chibotaru, A. Ceulemans, V. Bruyndoncx and V. V Moshchalkov. Vortex entry and nucleation of antivortices in a mesoscopic superconducting triangle. Phys. Rev. Lett. 86, 1323 (2001)

${ }^{15}$ L. F. Chibotaru, A. Ceulemans, G. Teniers, and V. V. Moshchalkov. Nucleation of superconductivity in regular polygons: Superconducting vector potential gauge approach. Physica C 369, 149 (2002).

16 L. F. Chibotaru, A. Ceulemans, V. Bruyndoncx, and V. V. Moshchalkov. Symmetry-induced formation of antivortices in mesoscopic superconductors. Nature 408, 833 (2000).

17 Kanda, B. J. Baelus, F. M. Peeters, K. Kadowaki and Y. Ootuka. Experimental evidence for giant vortex states in a mesoscopic superconducting disk. Phys. Rev. Lett. 93, 257002 (2004).

18 D. Song, et al. Unveiling pseudospin and angular momentum in photonic graphene. Nat. Commun. 6, 6272(2015).

19 J. Y. Ge, J. Gutierrez, V. N. Gladilin, J. T. Devreese and V. V. Moshchalkov. Bound vortex dipoles generated at pinning centres by Meissner current. Nat. Commun. 6, 6573 (2015).

20 F. S. Wells, A. V. Pan, X. R. Wang, S. A. Fedoseev and S. A. Hilgenkamp. Analysis of low-field isotropic vortex glass containing vortex groups in $\mathrm{YBa}_{2} \mathrm{Cu}_{3} \mathrm{O}_{7-\mathrm{x}}$ thin films visualized by scanning SQUID microscopy. Sci. Rep. 5, 8677 (2015).

21 A. Slachmuylders, B. Partoens, and F. M. Peeters. Geometry and magnetic-field-induced vortices and antivortices in mesoscopic two-dimensional systems. Phys. Rev. B 71, 245405 (2005).

22 O. Olendski and L. Mikhailovska. Analytical and numerical study of a curved planar waveguide with combined Dirichlet and Neumann boundary conditions in a uniform magnetic field. Phys. Rev. B 77, 174405 (2008).

23 C. S. Lent. Edge states in a circular quantum dot. Phys. Rev. B 43, 4179 (1991).

${ }^{24}$ W.-d. Sheng, M. Korkusinski, A. D. Güçlü, M. Zielinski, P. Potasz, E. S. Kadantsev, O. Voznyy, P. Hawrylak. Electronic and optical properties of semiconductor and graphene quantum dots. Front. Phys. 7(3), 328 (2012).

25 J. Ketterson and S. Song. Superconductivity. Cambridge University Press, 1999.

${ }^{26}$ K. S. Novoselov, A. K. Geim, S. V. Morozov, D. Jiang, Y. Zhang, S. V. Dubonos, I. V. Grigorieva, and A. A. Firsov. Electric Field Effect in Atomically Thin Carbon Films. Science 306,666 (2004).

${ }^{27}$ K. S. Novoselov, D. Jiang, F. Schedin, T. J. Booth, V.
V. Khotkevich, S. V. Morozov, and A. K. Geim. Twodimensional atomic crystals. Proc. Natl. Acad. Sci. U.S.A. 102, 10451 (2005).

28 A. K. Geim and K. S. Novoselov. The rise of graphene. Nature Mater. 6, 183 (2007).

29 A. H. Castro Neto, F. Guinea, N. M. R. Peres, K. S. Novoselov, and A. K. Geim. The electronic properties of graphene. Rev. Mod. Phys. 81, 109 (2009).

30 M. Ezawa. Dirac fermions in a graphene nanodisk and a graphene corner: Texture of vortices with an unusual winding number. Phys. Rev. B 81, 201402 (2010).

31 R. Peierls. On the theory of the diamagnetism of conduction electrons. Z. Physik 80, 763 (1933).

32 S. Schnez, K. Ensslin, M. Sigrist, and T. Ihn. Analytic model of the energy spectrum of a graphene quantum dot in a perpendicular magnetic field. Phys. Rev. B 78, 195427 (2008).

33 M. Grujić, M. Zarenia, A. Chaves, M. Tadić, G. A. Farias, and F. M. Peeters. Electronic and optical properties of a circular graphene quantum dot in a magnetic field: Influence of the boundary conditions. Phys. Rev. B 84, 205441 (2011).

34 M. Grujić, M. Zarenia, M. Tadić and F. M. Peeters. Interband optical absorption in a circular graphene quantum dot. Phys. Scr. T149, 014056 (2012).

35 M. Zarenia, A. Chaves, G. A. Farias, and F. M. Peeters. Energy levels of triangular and hexagonal graphene quantum dots: A comparative study between the tight-binding and Dirac equation approach. Phys. Rev. B 84, 245403 (2011).

${ }^{36}$ M. Zarenia, J. Milton Pereira, A. Chaves, F. M. Peeters, and G. A. Farias. Simplified model for the energy levels of quantum rings in single layer and bilayer graphene. Phys. Rev. B 81, 045431 (2010).

37 M. Ramezani Masir, A. Matulis, and F. M. Peeters. Scattering of Dirac electrons by circular mass barriers: Valley filter and resonant scattering. Phys. Rev. B 84, 245413 (2011).

38 L. J. P. Xavier, D. R. da Costa, A. Chaves, J. M. Pereira Jr., and G. A. Farias. Electronic confinement in graphene quantum rings due to substrate-induced mass radial kink. J. Phys.: Condens. Matter 28, 505501 (2016).

39 D. R. da Costa, Andrey Chaves, M. Zarenia, J. M. Pereira Jr., G. A. Farias, and F. M. Peeters. Geometry and edge effects on the energy levels of graphene quantum rings: A comparison between tight-binding and simplified Dirac models. Phys. Rev. B 89, 075418 (2014).

40 D. R. da Costa, M. Zarenia, Andrey Chaves, G. A. Farias, F. M. Peeters. Analytical study of the energy levels in bilayer graphene quantum dots. Carbon 78, 392 (2014).

41 D. R. da Costa, M. Zarenia, Andrey Chaves, G. A. Farias, F. M. Peeters. Magnetic field dependence of energy levels in biased bilayer graphene quantum dots. Phys. Rev. B 93, 085401 (2016).

42 M. Mirzakhani, M. Zarenia, D. R. da Costa, S. A. Ketabi, and F. M. Peeters. Energy levels of ABC-stacked trilayer graphene quantum dots with infinite-mass boundary conditions. Phys. Rev. B 94, 165423 (2016).

${ }^{43}$ E. A. de Andrada e Silva. Probability current in the tightbinding model. Am. J. Phys. 60, 8 (1992).

44 D. R. da Costa, A. Chaves, G. A. Farias, L. Covaci, and F. M. Peeters. Wave-packet scattering on graphene edges in the presence of a pseudomagnetic field. Phys. Rev. B 86, 115434 (2012). 
45 D. R. da Costa, Andrey Chaves, M. Zarenia, J. M. Pereira Jr., G. A. Farias, and F. M. Peeters. Geometry and edge effects on the energy levels of graphene quantum rings: A comparison between tight-binding and simplified Dirac models. Phys. Rev. B 89, 075418 (2014).

46 M. Governale and C. Ungarelli. Gauge-invariant grid discretization of the Schrödinger equation. Phys. Rev. B 58, 7816 (1998).

47 M. V. Berry and R. J. Mondragon. Neutrino billiards: Time-reversal symmetry-breaking without magnetic fields. Proc. R. Soc. Lond. Ser. A 412, 53 (1987).

48 Y. Liu, M. Brada, E. J. Mele, and F. V. Kusmartsev. Magnetisation oscillations, boundary conditions and the Hofstadter butterfly in graphene flakes. Ann. Phys. (Berlin) 526, 449 (2014).

${ }^{49}$ H. P. Heiskanen, M. Manninen, J. Akola. Electronic structure of triangular, hexagonal and round graphene flakes near the Fermi level. New J. Phys. 10, 103015 (2008).

50 Z. Z. Zhang, Kai Chang, and F. M. Peeters. Tuning of energy levels and optical properties of graphene quantum dots. Phys. Rev. B 77, 235411 (2008).
51 For negative integer values, i. e. $\beta=0,-1,-2, \ldots=-n$, the regularized confluent hypergeometric function can be written as $\widetilde{M}(\alpha,-n, z)=(a)_{n+1} z^{n+1} M(\alpha, \beta, z) /(n+1)$ !, where $(a)_{n+1}$ are the Pochhammer symbols.

${ }^{52}$ In our calculation we used the relation $d \widetilde{M}(\alpha, \beta, z) / d z=$ $\alpha \widetilde{M}(\alpha+1, \beta+1, z)$.

53 A. Matulis, F. M. Peeters. Quasibound states of quantum dots in single and bilayer graphene. Phys. Rev. B 77, 115423 (2008).

54 A. V. Rozhkov, and Franco Nori. Exact wave functions for an electron on a graphene triangular quantum dot. Phys. Rev. B 81, 155401 (2010).

55 D. R. da Costa, M. Zarenia, Andrey Chaves, G. A. Farias, and F. M. Peeters. Energy levels of bilayer graphene quantum dots. Phys. Rev. B 92, 115437 (2015).

${ }^{56}$ L. Graftieaux, M. Michard and N. Grosjean. Combining PIV, POD and vortex identification algorithms for the study of unsteady turbulent swirling flows. Meas. Sci. Technol. 12, 1422 (2001). 